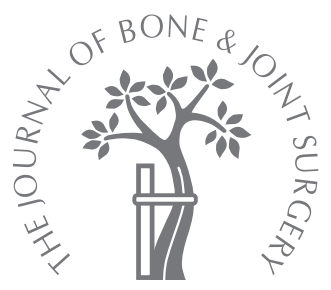

- CASE REPORT

\title{
Subcutaneous anthrax in three intravenous drug users
}

\author{
A NEW CLINICAL DIAGNOSIS
}

\begin{abstract}
D. Knox, G. Murray, M. Millar, D. Hamilton, M. Connor, R. D. Ferdinand, G. A. Jones

From the Dumfries and Galloway Royal Infirmary, Dumfries, United Kingdom
\end{abstract}

D. Knox, MRCS, Orthopaedic Registrar

I. Murray, MBChB, Orthopaedic House Officer - R. D. Ferdinand, FRCS(Tr\&Orth), Consultant Orthopaedic Surgeon

Department of Orthopaedics

M. Millar, MBChB, Medical House Officer

G. A. Jones, MRCP, DTM\&H, Consultant Physician \& Infectious Diseases Specialist Department of Medicine

D. Hamilton, MRCP, FRCPath

Consultant Microbiologist

= M. Connor, MRCP, FRCPath,

Consultant Microbiologist

Dumfries \& Galloway Royal

Infirmary, Bankend Road,

Dumfries DG1 4AP, UK.

Correspondence should be sent to Mr D. Knox; e-mail: davidknox@doctors.org.uk

(C)2011 British Editorial Society of Bone and Joint Surgery doi:10.1302/0301-620X.93B3. $25976 \$ 2.00$

$J$ Bone Joint Surg [Br] 2010;93-B:414-17.

Received 28 September 2010; Accepted 3 November 2010

\begin{abstract}
Anthrax is extremely rare in the western world but is endemic to areas of south and central Asia. In early 2010 an outbreak was identified in heroin-injecting intravenous drug users in the United Kingdom and Europe. Afghanistan is currently the principal source of heroin which reaches the United Kingdom. When anthrax occurs, cutaneous disease accounts for over $\mathbf{9 5 \%}$ of cases. At least 47 cases with 13 deaths have been confirmed so far. We present three cases presenting during this time with marked swelling, one resulting in compartment syndrome but all with an absence of the expected cutaneous appearances.

We suggest that rather than cutaneous anthrax, these patients represent a new subcutaneous presentation of anthrax.
\end{abstract}

Naturally acquired anthrax occurs typically in Africa and central and southern Asia. ${ }^{1}$ It is extremely rare in Western countries. Cutaneous disease accounts for over $95 \%$ of cases. ${ }^{2}$ The name anthrax is derived from the Greek for 'burnt coal'. The classic presentation progresses from painless vesicles and papules that burst but release no pus and produce a characteristic black eschar, ${ }^{2,3}$ accompanied by marked swelling (Fig. 1). A minority of patients, if untreated, will develop systemic anthrax which has a considerable mortality rate. ${ }^{1,4}$

In early 2010 an outbreak of anthrax was identified in heroin-injecting intravenous drug users in the United Kingdom and Europe. At least 47 cases with 13 deaths have been confirmed so far. ${ }^{5}$ There are only two reports of anthrax being transmitted via injection. ${ }^{6,7} \mathrm{We}$ describe three cases who presented to our hospital during this time with marked swelling but without the expected cutaneous appearances. We suggest that rather than cutaneous anthrax, these patients represent a new subcutaneous presentation. All patients were hepatitis $\mathrm{B}, \mathrm{C}$ and HIV negative.

\section{Case reports}

Case 1. A 44-year-old male intravenous drug user presented with a 48-hour history of increasing pain and swelling of his right arm, ten days after injecting into his antecubital fossa. He had compartment syndrome with a tense forearm, pain on passive movement of his fingers and paraesthesiae in his thumb. A small area of erythema distal to the injection site extended laterally toward the olecranon. He was apyrexial. Intravenous benzylpenicillin and flucloxacillin were administered prior to urgent decompression of the forearm and hand compartments with extensive fasciotomies. No necrotic muscle was evident. A thrombosed antecubital vein was tied off and a separate incision made over the olecranon. Free serous fluid was found at both incisions. Pus was not evident but post-operative oozing was considerable. After 48 hours the wounds on the hand, dorsal aspect of the forearm and olecranon were closed. An elastic vascular vessel loop was used to approximate the volar wound with a further return to theatre after 48 hours. Copious serous fluid was present throughout the wound, with marked oedema. Areas of the forearm superficial muscles had sluggish contracture and bled only upon incision (Fig. 2). The blood results are summarised in Table I.

On the fifth day after admission, a preliminary tissue culture was positive for Bacillus anthracis. Until then, gram stain and culture had revealed no organisms, no pus cells and no growth. Intravenous ciprofloxacin, benzylpenicillin and clindamicin were commenced. A national reference laboratory confirmed the identification of the organism on the following day using polymerase chain reaction on tissue from the wound. On the sixth day, three small haemorrhagic areas were evident in the axilla and the chest wall became erythematous and oedematous. Further debridement was neces- 


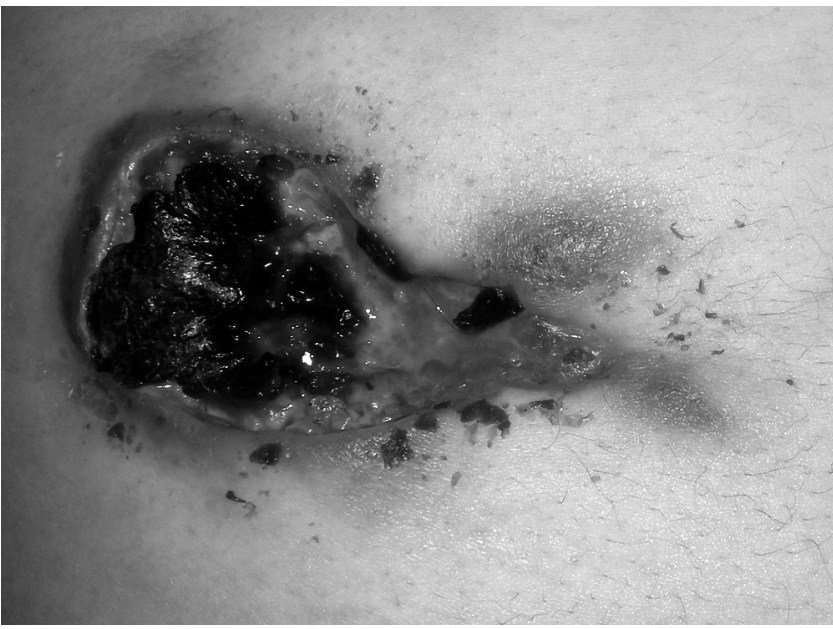

Fig. 1

Photograph showing a lesion on the abdominal wall with the characteristic features of cutaneous anthrax. The patient was an intravenous drug user and denied injecting at this site. The details of this patient are not reported here.

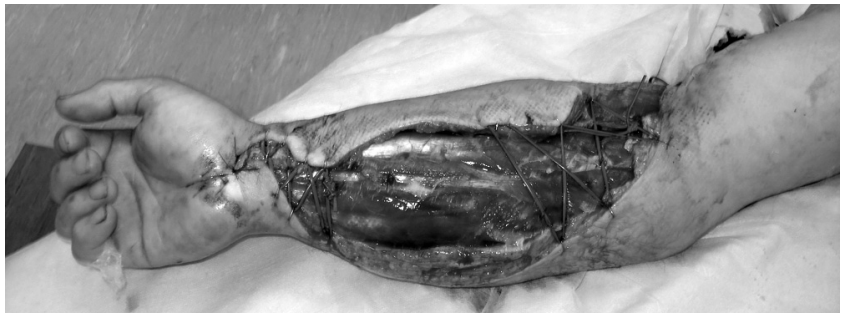

Fig. 2a

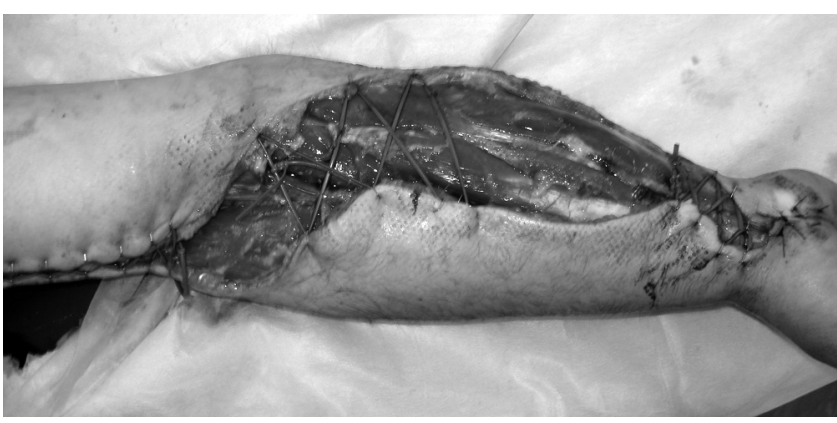

Fig. 2b

Case 1: photographs on the third visit to theatre showing increased swelling, serous discharge and oedema. The tension in the vascular loops is obvious.

sary, and he was transferred to a supraregional centre for plastic surgery. He remained haemodynamically stable but markedly hypoalbuminaemic. He developed multi-organ failure requiring renal dialysis and ventilator support. He was discharged after 60 days of in-patient care.
Table I. Case 1: biochemistry and haematology results during the initial six days after presentation

\begin{tabular}{lcccccc}
\hline \multicolumn{7}{c}{ Day after admission } \\
\cline { 2 - 7 } & $\mathbf{1}$ & $\mathbf{2}$ & $\mathbf{3}$ & $\mathbf{4}$ & $\mathbf{5}$ & $\mathbf{6}$ \\
\hline Sodium $(\mathrm{mmol} / \mathrm{L})$ & 136 & 125 & 131 & 121 & 117 & 117 \\
Creatinine $(\mu \mathrm{mol} / \mathrm{L})$ & 92 & 97 & 122 & 92 & 76 & 72 \\
WCC $^{*}\left(\times 10^{9} / \mathrm{L}\right)$ & 9.5 & 8.7 & 12.6 & 13.5 & 18.3 & 18.9 \\
CRP $(\mathrm{mg} / \mathrm{L})$ & 17 & 33 & 35 & 41 & 67 & 88 \\
Albumin $(\mathrm{g} / \mathrm{L})$ & - & - & - & - & - & 16 \\
$\mathrm{CK}^{\dagger}(\mathrm{IU} / \mathrm{L})$ & - & - & - & - & - & 868 \\
\hline * WCC, white cell count & & & & & \\
$+\mathrm{CK}$, creatine kinase & & & & & &
\end{tabular}

Case 2. A 36-year-old male intravenous drug user was referred from police custody with a swollen left forearm and hand having injected heroin into his antecubital fossa and forearm on the previous three days. He had no cutaneous stigmata of anthrax and was apyrexial. Finger movements were painful and restricted. There was considerable swelling but no compartment syndrome. A central line was established, blood cultures obtained and he was given intravenous ciprofloxacin, clindamicin, benzylpenicillin and metroniadazole. He underwent pre-emptive fasciotomy of his left hand, carpal tunnel and forearm compartments. All revealed marked free fluid and oedema. Multiple tissue samples were obtained and 48 hours later the hand and dorsal forearm wounds were closed and an elasticated vascular loop used to approximate the volar skin edges which were left open. In subsequent visits to theatre, increasing free fluid was present. There was no pus or necrotic muscle but the wound was left open to allow further inspection. He attended theatre on seven occasions before it was appropriate to close the wound with a split skin graft. There was excellent healing of the wound and gradual improvement in hand function.

Tissue samples tested positive by polymerase chain reaction for Bacillus anthracis but gram stain revealed only scanty pus cells and the culture was negative. Throughout the admission he remained apyrexial. His white cell count and CRP never rose above $9.1 \times 10^{9} / \mathrm{L}$ and $9 \mathrm{mg} / \mathrm{L}$ respectively, and sodium and albumin levels remained normal.

Case 3. A 32-year-old female intravenous drug user was referred with suspected abscesses in both lower limbs, with spreading cellulitis and oedema that had not responded to two days of oral flucloxacillin. She was apyrexial and systematically well. She had injected into the lateral aspects of both calves almost three weeks previously. An accidental blow to the right side had caused the lesions to become painful. These appeared to be typical injection abscesses, but debridement revealed a central necrotic plug surrounded by discoloured, liquefying fat. Within 24 hours, the diagnosis of anthrax was confirmed on four tissue samples by polymerase chain reaction. Meanwhile, it was noted that the dorsal aspect of both hands was swollen. This did not restrict movement and was not associated with 


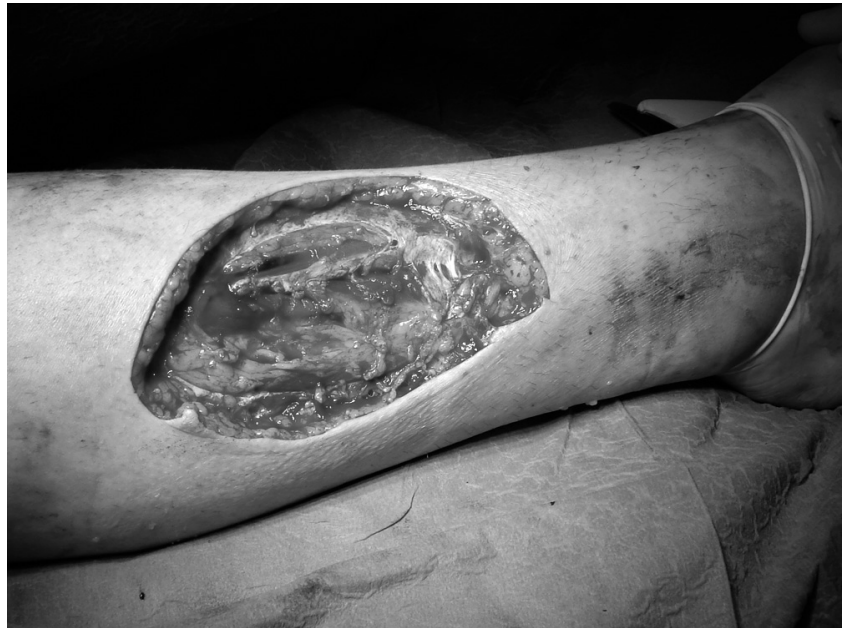

Fig. 3

Case 3: intra-operative photograph showing the extensive debridement required to excise the liquefied and necrotic fat.

paraesthesiae, a change in capillary refill, cellulitis or the formation of an abscess. However, the guttering between the metacarpophalangeal joints was indistinct and there was asymmetry between the hands. She had injected drugs subcutaneously on the palmar surface approximately three weeks previously. Debridement of both hands revealed fat necrosis and fibrosis with free fluid, and a small area of muscle necrosis in one lumbrical on the left side. These were inspected and washed out on another occasion before primary closure of the hands and split-skin graft closure of the lower limbs was performed (Fig. 3). Pre-operative wound swabs and blood cultures were negative to culture, polymerase chain reaction and gram stain. However, tissue samples from the lower limbs were polymerase chain reaction positive with leg wound swabs in theatre also culturing Bacillus anthracis. Clinically we believe that both hands reflected the now typical appearance of subcutaneous anthrax infection. Blood tests revealed that the white cell count and CRP did not exceed $7.4 \times 10^{9} / \mathrm{L}$ and $6 \mathrm{mg} / \mathrm{L}$, respectively. Sodium levels remained normal throughout. Post-operative discharge from the hand wounds required many changes of dressings.

\section{Discussion}

Bacillus anthracis belongs to a genus of encapsulated, aerobic, spore-forming rods, and spores can be dormant in the environment for many years. Once in favourable conditions for replication the spore is able to germinate into active bacteria. Bacillus anthracis produces two toxins, the oedema factor predominating in early infection, and the lethal factor emerging later. ${ }^{8}$

Anthrax is uncommon in the west. It is typically spread by contact with infected animals and their products, and modern veterinary practices have made this unlikely. It is, however, found in south and central Asian countries including Afghanistan, which is currently the principal source of illegal heroin in the United Kingdom.

There is only one previously reported case of anthrax transmission in an intravenous drug user. ${ }^{7}$ This case also records a lack of typical cutaneous manifestations, a delayed diagnosis and massive oedema. The patient eventually died from anthrax meningitis. There is also a report from India which first described transmission by injection. ${ }^{6}$ A 55 -year-old female received an injection of antibiotic into her right arm. After 24 hours, she developed localised swelling and tenderness and subsequently extensive swelling affecting the whole of the right upper limb, chest wall and neck. The injection site was incised, producing haemorrhagic, gelatinous material but not pus. Culture eventually revealed Bacillus anthracis. The authors speculated that as the patient came from an area endemic for anthrax, perhaps the skin was contaminated with spores which were inoculated into the subcutaneous layer at the time of injection.

The typical cutaneous manifestations of naturally acquired anthrax are well documented. In a review of 32 cases, Oncül et $\mathrm{al}^{9}$ recorded swelling, redness and eschar formation in all patients. Examples of recent images following the 2001 postal outbreak in the United States and from this episode involving intravenous drug users in the United Kingdom showed the formation of vesicles and erythaema surrounding cutaneous lesions. ${ }^{10,11}$

The role of surgical as opposed to medical management in this current outbreak is difficult to define. Compartment syndrome requires fasciotomy and the tissue samples from theatre were the only positive results in two of the three patients, whether by polymerase chain reaction or tissue culture analysis.

In conclusion, we report the first documented case of subcutaneous anthrax presenting as compartment syndrome. All of our cases occurred in heroin-injecting drug users. We suggest that swelling, serous discharge and oedema should be considered as potential signs of subcutaneous anthrax in intravenous drug users and the use of polymerase chain reaction testing to facilitate diagnosis. We believe that this cluster of symptoms, signs, laboratory and theatre findings may be characteristic of early infection with Bacillus anthracis acquired by subcutaneous or intravenous inoculation. It may therefore represent a new form of subcutaneous Bacillus anthracis infection without the typical cutaneous features.

No benefits in any form have been received or will be received from a commercial party related directly or indirectly to the subject of this article.

\section{References}

1. No authors listed. Anthrax in humans and animals. Fourth ed. Geneva: World Health Organization, 2008.

2. Swartz MN. Recognition and management of anthrax: an update. N Eng/ J Med 2001;345:1621-6.

3. Doganay L, Welsby PD. Anthrax: a disease in waiting? Postgrad Med J 2006;82:754-6. 
4. Holty JE, Bravata DM, Liu H, et al. Systematic review: a century of inhalational anthrax cases from 1900 to 2005. Ann Intern Med 2006;144:270-80.

5. No authors listed. Health Protection Scotland. Anthrax outbreak information. http:/ /www.hps.scot.nhs.uk/anthrax/index.aspx (date last accessed 27 September 2010).

6. Lalitha MK, Anandi V, Walter N, Devadatta J0, Pulimood BM. Primary anthrax presenting as an injection "abscess". Indian J Pathol Microbiol 1988;31:254-6.

7. Ringertz SH, Høiby EA, Jensenius $\mathbf{M}$, et al. Injectional anthrax in a heroin skinpopper. Lancet 2000;356:1574-5.
8. Sherer K, Li Y, Cui X, Eichacker PO. Lethal and edema toxins in the pathogenesis of Bacillus anthracis septic shock: implications for therapy. Am J Respir Crit Care Med 2007:175:211-21.

9. Oncül 0, Ozsoy MF, Gul HC, et al. Cutaneous anthrax in Turkey: a review of 32 cases. Scand J Infect Dis 2002;34:413-16.

10. Stevenson J, Mardon J, Exton A. Minerva. BMJ 2010;340:c889.

11. Roche JK, Chang MW, Lazar H. Images in clinical medicine: cutaneous anthrax infection. N Engl J Med 2001;345:1611. 